\title{
Four-Generation Low Energy Supersymmetry with a Light Top Quark
}

\author{
M. Carena, H.E. Haber ${ }^{\star}$ and C.E.M. Wagner \\ CERN, TH Division, \\ CH-1211 Geneva 23, Switzerland
}

\begin{abstract}
A supersymmetric model with four generations is proposed, in which the top quark is approximately degenerate in mass with the $W^{ \pm}$gauge boson, $m_{t} \simeq m_{W}$, leading to values of $R_{b}$ in better agreement with the present experimental data than in the Standard Model. The model shares many of the good features of the minimal supersymmetric extension of the Standard Model (MSSM), such as the unification of gauge and Yukawa couplings at a common high-energy scale. The model differs from the MSSM by re-interpreting the Tevatron "top-quark" events as the production of the fourth generation quark $t^{\prime}$, which decays dominantly to $b W^{+}$. The top quark decays primarily into supersymmetric particles, $t \rightarrow \tilde{t} \tilde{\chi}_{1}^{0}$, with $\tilde{t} \rightarrow$ $c \tilde{\chi}_{1}^{0}$, thereby evading previous searches. Light supersymmetric particles are predicted to lie in the mass range between 25 and $70 \mathrm{GeV}$, which together with the fourth generation leptons provide a rich spectrum of new physics which can be probed at LEP-2 and the Tevatron.
\end{abstract}

CERN-TH/95-311

December 1995

* Permanent address: Santa Cruz Institute for Particle Physics, University of California, Santa Cruz, CA 94064 USA. 
Recently, the CDF and D0 Collaborations have announced the discovery of the top quark at the Tevatron [1], with a measured mass of $m_{t}=176 \pm 8 \pm 10 \mathrm{GeV}$ and $m_{t}=199_{-21}^{+19} \pm 22 \mathrm{GeV}$, respectively. Both measurements are in excellent agreement with the top quark mass deduced by the LEP global analysis of precision electroweak measurements. The most recent numbers quoted by the LEP Electroweak Working Group from a Standard Model fit with a Higgs mass of $300 \mathrm{GeV}$ are: $m_{t}=170 \pm$ $10_{-19}^{+17} \mathrm{GeV}\left[180_{-9}^{+8}+17 \mathrm{GeV}\right]$ based on the global average of the data from the four LEP detectors and excluding [including] data from the SLD Collaboration [2], where the last positive and negative error quoted corresponds to alternative Higgs mass choices of $1 \mathrm{TeV}$ and $60 \mathrm{GeV}$, respectively. The LEP determination of $m_{t}$ derives from the sensitivity of electroweak observables in $Z$ decays to virtual top quark exchange, which enters in two distinct ways. First, top quark loops in gauge boson self-energies (the so-called oblique corrections) can directly affect the properties of the $Z$ and $W^{ \pm}$. The most important oblique correction is the top-quark contribution to the electroweak $\rho$ parameter [3], which is given by $\rho=1+\Delta \rho$, where $\Delta \rho \simeq 3 G_{F} m_{t}^{2} / 8 \pi^{2} \sqrt{2}$. Second, top-quark loops can contribute to three-point (and higher) interactions (these are the non-oblique corrections). The most significant non-oblique correction to $Z$ decays arises in the vertex correction to $Z \rightarrow b \bar{b}$, which is also quadratically sensitive to the top quark mass. The size of the oblique corrections, which can be inferred from the present precision data analysis, is in very good agreement with the Standard Model theoretical expectations. In contrast, the measured value of the rate for $R_{b} \equiv \Gamma(Z \rightarrow$ $b \bar{b}) / \Gamma(Z \rightarrow$ hadrons $)$ lies more than three standard deviations above the Standard Model prediction.

If the deviation in the observed value of $R_{b}$ holds under further experimental scrutiny, then this would be a signal of new physics beyond the Standard Model, arising (presumably) from the virtual exchange of new particles. In the literature, models of low-energy supersymmetry have been examined in which $R_{b}$ is slightly enhanced above the Standard Model prediction [4-13]. In such models, the global fit to the electroweak data is improved. Note that in order to improve the Standard Model fit, one must approximately maintain the size of the Standard Model oblique corrections while modifying the $Z b \bar{b}$ interaction. In the minimal supersymmetric extension of the Standard Model (MSSM), this is possible if one takes large [small] values of the mass parameters of the scalar super-partners of the left [right] handed top quark, and small values of the Higgs superfield mass parameter $\mu$.

In this paper, we take an alternative approach. If one considers the $R_{b}$ measurement alone within the context of the Standard Model, one would conclude that $m_{t} \lesssim m_{W}$. To avoid conflict with the measurement of $m_{t}$ at the Tevatron (by direct means) and at LEP (by indirect means) as described above, one must invoke new physics to explain the CDF and D0 "top-quark" events and new virtual effects to generate the oblique radiative corrections observed in precision electroweak experiments. In addition, one must explain why such a light top-quark has escaped detection in previous experiments at hadron colliders. Surprisingly, a model with such a light top-quark exists which is not in conflict with present experimental observations. The model consists of the 
nearly $100 \%$ branching ratio (where $\tilde{\chi}_{1}^{0}$ is the lightest neutralino, assumed to be the lightest supersymmetric particle). The CDF and D0 "top-quark" events are identified as $t^{\prime} \rightarrow b W^{+}$, and the oblique radiative corrections observed in precision electroweak data are approximately saturated by loops of third and fourth generation quarks and squarks.

In section 2 we review in more detail the top-quark mass dependence of precision electroweak observables, and discuss the implications of the recent measurement of $R_{b}$ (and the corresponding measurement of $R_{c}$ ). We discuss some of the requirements for the viability of the light top quark explanation of the observed discrepancy in $R_{b}$. In section 3 we analyze the restrictions on the fourth generation masses due to a variety of phenomenological and theoretical constraints. By imposing the requirement that none of the couplings of the model become nonperturbative below the grand unification (GUT) scale, we find that all fourth generation fermion-Higgs Yukawa couplings approximately unify at the GUT scale while approaching quasi-infrared fixed point values at the electroweak scale. In sections $4-6$ we provide a detailed phenomenological profile of our model, and discuss the constraints on its parameters, with particular attention given to the implications of the precision electroweak data and flavor changing processes. Section 7 summarizes the successes and the shortcomings of our model. Some technical details are included in the Appendix. A preliminary version of this work was presented in Ref. [14].

\section{The top quark mass from precision electroweak data}

Experiments at LEP and SLC measure more than fifteen separate electroweak observables in $Z$ decay events. Global fits of these observables exhibit a remarkable consistency with Standard Model expectations, with two notable exceptions. Defining $R_{q} \equiv \Gamma(Z \rightarrow q \bar{q}) / \Gamma(Z \rightarrow$ hadrons $)$, with $q=b, c$, the LEP Electroweak Working Group global fit yields $[2]$

$$
R_{b}= \begin{cases}0.2219 \pm 0.0017, & \text { LEP } / \text { SLC global fit } \\ 0.2156, & \text { Standard Model prediction }\end{cases}
$$

which is a $3.7 \sigma$ discrepancy, and

$$
R_{c}= \begin{cases}0.1543 \pm 0.0074, & \text { LEP/SLC global fit } \\ 0.1724, & \text { Standard Model prediction }\end{cases}
$$

which is a $2.5 \sigma$ discrepancy. One other LEP measurement relevant to this discussion is the $\alpha_{s}\left(m_{Z}\right)$ determination $[9,15-17]$ from the total hadronic width of the $Z$. Based on the measurement of $R_{\ell} \equiv \Gamma_{\text {had }} / \Gamma_{\ell \ell}$, the Standard Model fit yields $[2] \alpha_{s}\left(m_{Z}\right)=$ $0.126 \pm 0.005 \pm 0.002$ (where the last error quoted corresponds to varying the Higgs mass from $60 \mathrm{GeV}$ to $1 \mathrm{TeV}$ ). It is interesting to note that the LEP determination of $\alpha_{s}\left(m_{Z}\right)$ tends to be somewhat higher than the extrapolated value of $\alpha_{s}\left(m_{Z}\right)$ obtained from lower energy measurements.

One must be very careful in interpreting the $R_{b}$ and $R_{c}$ discrepancies from Standard Model expectations. The experimental procedures that identify $b$ and $c$ quarks in $Z$ 
ment, we note first that the quoted error is larger, and the statistical significance of the deviation from the Standard Model prediction is smaller, than those of $R_{b}$. Second, it is very difficult to imagine new physics processes that could produce such a large deviation in $R_{c}$. To understand the latter point, suppose new physics were responsible for both the $R_{b}$ and $R_{c}$ discrepancies. Because $R_{b}+R_{c}$ is less than the Standard Model prediction, one is led to a distressing conclusion. Since $\alpha_{s}\left(m_{Z}\right)$ is determined from the experimentally measured $\Gamma_{\text {had }}$, a negative contribution of new physics to this quantity implies that the perturbative QCD corrections to $\Gamma_{\text {had }}$ should be larger. That is, $\alpha_{s}\left(m_{Z}\right)$ determined from $\Gamma_{\text {had }}$ would be larger than the number quoted above, in significant conflict with the low-energy determinations. This argument implicitly assumes that there are no new physics contributions to $R_{q}$ where $q$ is a light quark. On the other hand, there is no known source of new physics that can modify $R_{q}$ sufficiently to compensate the deficit in $R_{b}+R_{c}$ to avoid the above conclusion. Thus, we are inclined to discount the measured value of $R_{c}$ above, and assume that its true value is close to the Standard Model expectation. Should we discount the measured value of $R_{b}$ as well? Further experimental analysis is required to clarify the situation. Here, we simply note that if new physics contributes to $R_{b}$ but not to $R_{c}$ and $R_{q}$, then because $R_{b}$ is above the Standard Model prediction, $\alpha_{s}\left(m_{Z}\right)$ determined from $\Gamma_{\text {had }}$ will be lower, potentially closer to the low energy $\alpha_{s}$ determinations [9,15-17].

Henceforth, we assume that $R_{c}$ is given by its Standard Model prediction. In the experimental determination of $R_{b}$, there is some contamination of $c \bar{c}$ events in the $b \bar{b}$ sample that must be subtracted. This subtraction depends on the value of $R_{c}$ assumed. Fixing $R_{c}$ to its Standard Model value, a slightly smaller value of $R_{b}$ is found by the Electroweak working group compared to the value quoted above:

$$
R_{b}=0.2205 \pm 0.0016, \quad \text { LEP/SLC global fit }
$$

roughly a three standard deviation discrepancy from the Standard Model prediction.

The $Z b \bar{b}$ vertex corrections can also significantly affect the left-right $b \bar{b}$ asymmetry $\mathcal{A}_{b}$,

$$
\mathcal{A}_{b}=\frac{g_{L}^{2}-g_{R}^{2}}{g_{L}^{2}+g_{R}^{2}}
$$

where $g_{L}\left(g_{R}\right)$ are the couplings of the left (right) handed bottom quarks to the $Z$. The corrections to $R_{b}$ and $\mathcal{A}_{b}$ can be parameterized as a function of the corrections to the left- and right-handed bottom quark vertices,

$$
\begin{gathered}
\frac{\delta \mathcal{A}_{b}}{\mathcal{A}_{b}}=\frac{4 f_{R} f_{L}}{f_{L}^{4}-f_{R}^{4}}\left[f_{R} \delta g_{L}-f_{L} \delta g_{R}\right], \\
\frac{\delta R_{b}}{R_{b}}=\frac{2\left(1-R_{b}\right)}{f_{L}^{2}+f_{R}^{2}}\left[f_{R} \delta g_{R}+f_{L} \delta g_{L}\right],
\end{gathered}
$$

where $f_{L}=1 / 2-\sin ^{2} \theta_{W}\left(m_{Z}\right) / 3$ and $f_{R}=-\sin ^{2} \theta_{W}\left(m_{Z}\right) / 3$ are the tree level couplings of the left and right handed bottom quarks to the $Z$. The dominant, top quark mass 
coupling to the $Z$,

$$
\delta g_{L}=-\frac{\alpha}{16 \pi \sin ^{2} \theta_{W}} m_{t}^{2}
$$

The large difference between the values of $f_{L}$ and $f_{R}$ implies that for $\delta g_{R}=0, \delta R_{b} / R_{b} \simeq$ $11.5 \delta \mathcal{A}_{b} / \mathcal{A}_{b}$. Moreover, the current determination of $\mathcal{A}_{b}$ at SLC is still subject to large experimental errors $[2]$

$$
\mathcal{A}_{b}=\left\{\begin{array}{ll}
0.841 \pm 0.053 & \text { LEP/SLC global fit; } \\
0.935, & \text { Standard Model prediction. }
\end{array} .\right.
$$

Therefore, $\mathcal{A}_{b}$ does not provide at present any significant constraint on the value of the top quark mass.

Barring additional experimental problems, and even after taking into account the possible systematic errors due to an incorrect charm background subtraction, the discrepancy in $R_{b}$ quoted in Eq. (2.3) is large enough as to raise concerns about the Standard Model interpretation of the data. As noticed above, if one extracts the prediction of the top quark mass from the $R_{b}$ measurement alone, one would conclude that the top quark is much lighter than the value inferred from the global LEP fit to the data or from the direct Tevatron measurements. Of course, with such a light top quark, one must address the obvious questions of why such a top quark has not yet been discovered, and what is the particle recently announced by the CDF and D0 Collaborations that is observed to decay into $b W^{+}$.

If the top quark were sufficiently light, then $W^{+} \rightarrow t \bar{b}$ would be kinematically allowed; this would modify the total width of the $W^{ \pm}$. But $\Gamma_{W}$ can be measured at hadron colliders indirectly by studying the ratio of production cross section times leptonic branching ratio of the $W^{ \pm}$and $Z$. The most recent analysis of this kind, reported by the CDF collaboration [18], finds $m_{t}>62 \mathrm{GeV}$ at $95 \% \mathrm{CL}$. (The most recent D0 data [19] implies a similar bound. A global average of all hadron collider data would raise this limit by a few $\mathrm{GeV}$.) Direct searches for the top quark at hadron colliders assume that an observable fraction of top quark decays results in a lepton in the final state. For example, the D0 collaboration ruled out the mass range $m_{W}+m_{b} \lesssim$ $m_{t}<131 \mathrm{GeV}$, assuming that the decay $t \rightarrow b W^{+}$is not unexpectedly suppressed [20]. Previous top quark searches at hadron colliders were able to close the window between $62 \mathrm{GeV}$ and $m_{W}+m_{b} \simeq 85 \mathrm{GeV}$, assuming that $t \rightarrow b W^{\star}$ is the dominant top-quark decay mode. However, in the latter case, the final state is three-body since $W^{\star}$ is virtual. If the top quark possessed any two-body decay modes (due to new physics processes), and if these modes rarely produced leptons, then a top quark in this mass region could have escaped detection in all previous top quark searches.

One possible scenario leading to two-body decay modes of the top quark arises if one assumes the existence of a light charged Higgs particle in the spectrum, $m_{H^{ \pm}}<$ $m_{W}$. Recent results from the CDF Collaboration rule out a substantial portion of the allowed parameter space [21] based on a direct search for $t \rightarrow b H^{+}$. Additional constraints arise from considering the contribution of virtual charged Higgs effects to heavy quark decays. In particular, one-loop charged Higgs exchange can significantly modify the predictions for rare bottom quark decay rates. Indeed, from the requirement 
this scenario is ruled out [22], unless the enhancement of this branching ratio due to the presence of a light charged Higgs boson is compensated by the contribution of new particles appearing at the electroweak scale.

Thus, we turn to an alternative scenario, based on low-energy supersymmetry with a light top-squark, in which the decay $t \rightarrow \tilde{t} \tilde{\chi}_{1}^{0}$ is kinematically allowed (where $\tilde{t}$ is the lightest top squark and $\tilde{\chi}_{1}^{0}$ is the lightest neutralino) [23]. Experimental searches for both $\tilde{t}$ and $\tilde{\chi}_{1}^{0}$ place constraints on their masses, but do not rule out the possibility of $M_{\tilde{t}}+M_{\tilde{\chi}_{1}^{0}}<m_{W}$. In particular, the LEP neutralino and chargino searches [24] imply a limit on the lightest neutralino mass which typically lies between 20 and $25 \mathrm{GeV}$ (although lighter neutralino masses cannot be ruled out if $\tan \beta$ is very close to one). Using this result and the limits on the top squark mass from searches at LEP and at the Tevatron [25], one finds that the mass region $50 \mathrm{GeV} \lesssim M_{\tilde{t}} \lesssim 60 \mathrm{GeV}$ (with $M_{\tilde{\chi}_{1}^{0}} \gtrsim 20 \mathrm{GeV}$ ) cannot be excluded. ${ }^{1}$

To be definite, we choose $m_{t} \simeq m_{W}, M_{\tilde{t}} \simeq 50 \mathrm{GeV}$ and $M_{\tilde{\chi}_{1}^{0}} \simeq 25 \mathrm{GeV}$. Then, the dominant decay chain is $t \rightarrow \tilde{t} \tilde{\chi}_{1}^{0}$ followed by $\tilde{t} \rightarrow c \tilde{\chi}_{1}^{0}$ through a one-loop process [23], which rarely produces a hard isolated lepton. Hence, these events would not have been detected at hadron colliders. But, now we must reconsider the recent CDF and D0 discoveries and the LEP "measurement" of $m_{t}$. As anticipated in section 1 , we propose to account for these results by introducing a fourth generation of quarks (and leptons) plus their supersymmetric partners. Then, $t^{\prime} \rightarrow b W^{+}$can be the source of the CDF and D0 events, while the effects of the third and fourth generation quarks and squarks contributing to the oblique corrections are large enough to be consistent with LEP precision electroweak data.

\section{Fourth generation fermion masses and unification}

For the $t^{\prime}$ to be consistent with the CDF and D0 "top-quark" events, its mass should be close to the world average, $m_{t^{\prime}}=180 \pm 12 \mathrm{GeV}$. We assume that the $t^{\prime}$ mass is given by $m_{t^{\prime}} \simeq 175 \mathrm{GeV}$. Moreover, in order to produce the observed Tevatron signal, the dominant $t^{\prime}$ decay must be $t^{\prime} \rightarrow b W^{+}$, in which the generation number changes by one unit. This means that two body generation number conserving decays, such as $t^{\prime} \rightarrow b^{\prime} W^{+}$or the supersymmetric decay modes $t^{\prime} \rightarrow \tilde{b^{\prime}} \tilde{\chi}^{ \pm}$and $t^{\prime} \rightarrow \tilde{t^{\prime}} \tilde{\chi}^{0}$, must be kinematically forbidden. Of course, three-body generation number conserving decays will be possible (e.g., $t^{\prime} \rightarrow b^{\prime} W^{*}$, where the virtual $W^{\star}$ decays in the usual way to a fermion antifermion pair). It follows that the $t^{\prime}-b$ mixing angle $\left(V_{t^{\prime} b}\right)$ must not be too small; otherwise the three-body generation conserving decays will dominate. For example, we find:

$$
\frac{\Gamma\left(t^{\prime} \rightarrow b^{\prime} W^{\star}\right)}{\Gamma\left(t^{\prime} \rightarrow b W\right)}=\frac{9 G_{F} m_{t^{\prime}}^{2}\left|V_{t^{\prime} b^{\prime}}\right|^{2}}{\pi^{2} \sqrt{2}\left|V_{t^{\prime} b}\right|^{2}} \int_{0}^{1-2 \sqrt{x}+x} \frac{z(1-z+x) \sqrt{(1-z+x)^{2}-4 x}}{\left(1-z m_{t^{\prime}}^{2} / m_{W}^{2}\right)^{2}} d z,
$$

\footnotetext{
${ }^{1}$ The most recent LEP run, at $\sqrt{s}=136 \mathrm{GeV}$, is not sensitive to this range of masses in the case of maximal top squark mixing (the latter is a necessary feature of our model).
} 
with the QCD prediction for $t \bar{t}$ production under the assumption that $\mathrm{BR}\left(t \rightarrow b W^{+}\right) \simeq$ 1 , a reinterpretation of these events as $t^{\prime} \bar{t}^{\prime}$ production (followed by $t^{\prime} \rightarrow b W^{+}$) requires $\operatorname{BR}\left(t^{\prime} \rightarrow b W^{+}\right)$to be near 1. At present, there are no relevant constraints on the mixing parameter $V_{t^{\prime} b}$. We assume that $V_{t^{\prime} b}$ lies between $V_{c b}=0.04$ and $V_{u s}=0.2$, although even larger values may be allowed in some circumstances [26]. For definiteness, we choose $\left|V_{t^{\prime} b} / V_{t^{\prime} b^{\prime}}\right|=0.1$.

In Figure 1, we show the dependence of the branching ratio $\operatorname{BR}\left(t^{\prime} \rightarrow b W^{+}\right)$on the $b^{\prime}$ mass. We see that there is a sharp increase of this branching ratio as soon as $m_{b^{\prime}}+m_{W} \gtrsim m_{t^{\prime}}$. Then, if we require $\operatorname{BR}\left(t^{\prime} \rightarrow b W^{+}\right) \gtrsim 0.75$ for the chosen value of $V_{t^{\prime} b}$, it follows that $m_{b^{\prime}} \gtrsim 105 \mathrm{GeV}$. Such a large value of the fourth generation down-type quark mass implies that the custodial SU(2) symmetry breaking is softer than in the three generation Standard Model case. As we shall discuss in section 5, this has important consequences for the value of the $\rho$-parameter. In particular, values of the $b^{\prime}$ mass too close to $175 \mathrm{GeV}$ would immediately imply a suppression of the oneloop contribution to the $\rho$-parameter, which is in conflict with the present precision electroweak data. Hence, the $b^{\prime}$ mass should either be larger than the $t^{\prime}$ mass or close to its experimental lower bound, $m_{b^{\prime}} \simeq 105 \mathrm{GeV}$. Having introduced a fourth generation of quarks, the simplest way to avoid electroweak gauge anomalies is to introduce a fourth generation of sequential leptons. In order to be consistent with present LEP-1 bounds, their masses must be larger than $45 \mathrm{GeV}$ (see discussion below).

90

$\mathrm{BR}\left(\mathrm{t}^{\prime} \rightarrow \mathrm{W}^{+} \mathrm{b}\right)$

Figure 1. Branching fraction $\mathrm{BR}\left(t^{\prime} \rightarrow b W^{+}\right)^{\circ} \mathrm{A} B R\left(\hat{t}^{\prime} \rightarrow b^{\prime} W^{+}\right)^{\circ}$ as a function of the fourth generation bottom mass $m_{b}$.

In the absence of any assumption about the nature of the physics at high energy scales, it is simple to construct a four-genezation model, which fulfills all the relevant experimental bounds, by simply pushing $\mathrm{u}_{\bar{y}}^{\mathrm{u}}$ the $b^{\prime}$ quark mass above the $t^{\prime}$ quark mass. In contrast, we find that our four-generation model is remarkably well-constrained if we

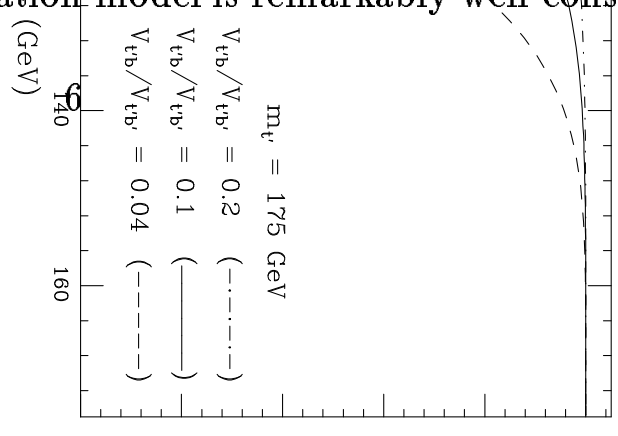


in the absence of grand unification, the Planck scale). In the latter case, the parameters of the model are almost uniquely determined, and the model is on the verge of being ruled out (or poised for experimental discovery).

One of the strongest motivations for low-energy supersymmetric extensions of the Standard Model comes from the fact that such models can be realized as effective low energy descriptions of theories in which all known low-energy gauge interactions unify at a high energy scale. In the MSSM, the values of the low energy gauge couplings and third generation fermion masses are consistent with the unification of gauge and Yukawa couplings at a single high energy scale $M_{\mathrm{GUT}} \simeq 2 \times 10^{16} \mathrm{GeV}[27]$. A fourth generation of fermions and their supersymmetric partners can be accommodated in complete $\mathrm{SU}(5)$ superfield multiplets, $\mathbf{1 0}+\overline{\mathbf{5}}$, and does not affect the successful $\sin ^{2} \theta_{W}\left(m_{Z}\right)$ prediction coming from the gauge coupling unification. The two-loop running of the gauge couplings is only slightly modified by the presence of the fourth generation, leading to a value of $\alpha\left(M_{\mathrm{GUT}}\right) \simeq 0.08$ for the unified gauge coupling and a slightly larger value of the unification scale, $M_{\mathrm{GUT}} \simeq 5 \times 10^{16} \mathrm{GeV}$. Moreover, taking $\alpha_{e m}\left(m_{Z}\right)$ and $\sin ^{2} \theta_{W}\left(m_{Z}\right)$ as input parameters, the two loop corrections enhance slightly the predicted values of $\alpha_{s}\left(m_{Z}\right)$ with respect to the value obtained in the MSSM [28]. This enhancement is partially suppressed by the effect of the large fourth generation Yukawa couplings, which happen to be close to their fixed point values. In any case, as in the MSSM, the predicted value of the strong gauge coupling, $\alpha_{s}\left(M_{Z}\right) \simeq 0.13$, is somewhat high to be consistent with the values of the strong gauge coupling extracted from LEP within the assumptions of this model. However, it is enough to assume the presence of small threshold corrections at the grand unification scale to produce a predicted value of $\alpha_{s}\left(M_{Z}\right)$ that is consistent with the present experimental data.

Consider next the requirement of perturbative consistency of the Yukawa sector of the theory up to scales of order $M_{\mathrm{GUT}}$. The low energy Higgs- $b^{\prime}$ quark Yukawa coupling is related to the running mass $m_{b}$, by

$$
h_{b^{\prime}}=\frac{\sqrt{2} m_{b^{\prime}}}{v \cos \beta},
$$

where $v=246 \mathrm{GeV}$ and $\tan \beta$ is the ratio of the two Higgs vacuum expectation values. For a fixed value of $m_{b}$, an upper bound on $\tan \beta$ may be hence obtained. For a physical mass $m_{b^{\prime}} \gtrsim 105 \mathrm{GeV}$, only small values of $\tan \beta<2$ are allowed. On the other hand, the Higgs- $t^{\prime}$ quark Yukawa coupling is related to its running mass by

$$
h_{t^{\prime}}=\frac{\sqrt{2} m_{t^{\prime}}}{v \sin \beta} .
$$

Consequently, low values of $\tan \beta$ tend to push the $t^{\prime}$ mass to relatively low values, unless its Yukawa coupling is itself close to its perturbativity bound, corresponding to the quasi-infrared fixed point value of this quantity. Large values of $h_{t}$, have the additional effect of changing the renormalization group evolution of $h_{b^{\prime}}$, leading to lower values of $m_{b^{\prime}}$ (or equivalently, lower values of $\tan \beta$ at fixed $m_{b^{\prime}}$ ). As a result, in order to obtain values of the fourth generation $b^{\prime}$ and $t^{\prime}$ masses consistent with the limits 
1. The $b^{\prime}$ mass must be close to its lower bound. Remarkably, this conclusion coincides with the one obtained from the considerations of the fourth generation contributions to the $\rho$-parameter, discussed in more detail in section 5 .

2. The $b^{\prime}$ and $t^{\prime}$ Yukawa couplings must lie close to their quasi infrared fixed point values $[29]$.

Therefore, the $b^{\prime}$ mass is essentially fixed by perturbativity arguments. Moreover, since we are close to the infrared fixed point values of the Yukawa couplings, $\tan \beta$ is also fixed. Figure 2 shows the resulting fourth generation quark masses as a function of $\tan \beta$ assuming that both Yukawa couplings lie close to their fixed point values. For $m_{t^{\prime}} \simeq 175 \mathrm{GeV}$ and $m_{b^{\prime}}=105 \mathrm{GeV}$; we obtain $\tan \beta \simeq 1.6$. Our picture is consistent with the unification of the $t^{\prime}$ and $b^{\prime}$ Yukawa couplings at $M_{\mathrm{GUT}}$ and, unlike the case of gauge coupling unification, the predicted low energy values are highly insensitive to possible moderate threshold corrections at $M_{\mathrm{GUT}}$.

Figure 2. Plot of the $b^{\prime}$ and $t^{\prime}$ quark masses as a function of $\tan \beta$ assuming that both lie close to their fixed point values.

The Tevatron may be able to rule out the existence of the $b^{\prime}$ with mass $m_{b^{\prime}} \simeq$ $105 \mathrm{GeV}$ (although the existing experimental limits only/rule out $b^{\prime}$ quarks of mass up to $85 \mathrm{GeV}$ [24]). If kinematically allowed, the decay $b^{\prime \prime} \rightarrow \tilde{t} \tilde{\chi}_{1}^{-}$would $\bar{b}$ e the dominant decay mode. If disallowed, there would be a competitión between $b^{\prime} \rightarrow W \epsilon$ (a change of two generations) and $b^{\prime} \rightarrow W^{\star} t$ (a change of one genération, but suppressed by threebody phase space). One option is to chळुose $\left|V_{b^{\prime} c}\right| \ll^{\prime}\left|V_{t^{\prime} b}\right|$ to remove the possibility of $b^{\prime} \rightarrow W c$. Then, all $b^{\prime}$ decays would result in the final state $W^{\star} c \tilde{\chi}_{1}^{0} \bar{\chi}_{1}^{0}$. - There are no published limits that exclude such a $b^{\prime}$. However, a dedicated search at the Tevatron

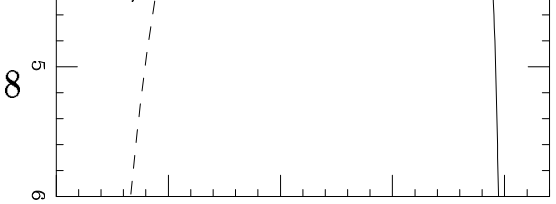


The fourth generation lepton Yukawa couplings are also strongly constrained by the experimental bound on the lepton masses $m_{\ell^{\prime}}>45 \mathrm{GeV}$. When applied to the neutrino mass, this bound implies that the right-handed Majorana mass, if present, must be of order of the electroweak scale. In addition, the bounds on the $\tau^{\prime}$ lepton mass imply that its Yukawa coupling must be larger than $\mathcal{O}(1)$ at the grand unification scale. We find that the infrared fixed point value for $m_{\tau^{\prime}}$ is very close to the current experimental bound on this quantity. Although there is more freedom in the $\tau^{\prime}-N_{\tau^{\prime}}$ sector, one can also add the requirement that both fourth generation Yukawa couplings take large values at $M_{\mathrm{GUT}}$ (in order to maximize their masses). Figure 3 shows the predicted values for the fourth generation lepton masses assuming that the neutrino $N_{\tau^{\prime}}$ is a Dirac neutrino, and that the four fourth-generation Yukawa couplings are close to their fixed point values. Then, for $\tan \beta \simeq 1.6$, the resulting lepton masses are: $m_{\tau^{\prime}} \simeq 50 \mathrm{GeV}$ and $m_{N_{\tau^{\prime}}} \simeq 80 \mathrm{GeV}$. It is interesting to note that these lepton masses are consistent with the unification of all four fermion-Higgs Yukawa couplings at the GUT scale. Moreover, these masses lie above the corresponding bounds from LEP-1, but both are in the kinematic reach of LEP-2.

90

$$
\mathrm{m}_{\mathrm{f}}[\mathrm{GeV}]
$$

Figure 3. The same as Fig. 2 but for the $\tau$ and the fourth generation neutrino masses.

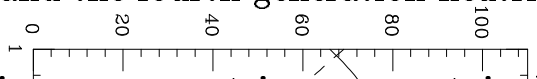

In general, the fourth generation neutrino mass matrix' can contain both an offdiagonal Dirac mass, $m_{D}$ and a right-handed-diagonal Majorana mass, $m_{M}$. Thus, in the more general case, the two fourth generâtion neustrino eigenstates are split by the see-saw mechanism. The lighter state has mass given by

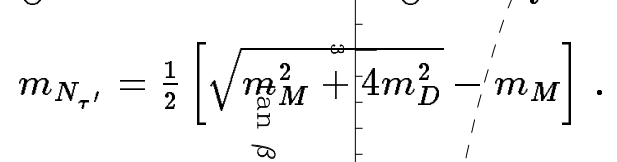

The infrared fixed point limit of $m_{D}$ coincides with that of $m_{N_{\tau}}$, shown in Figure 3 . There is no corresponding limit on the Majorana'mass $m_{M}$. For $m_{D} \approx 80 \mathrm{GeV}$, it

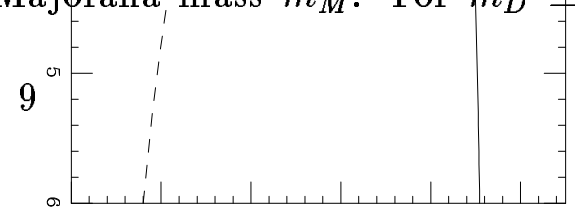


state becomes approximately degenerate with the $\tau^{\prime}$. This observation implies that a range of parameters exists where $N_{\tau}$, is a few $\mathrm{GeV}$ lighter than the $\tau^{\prime}$. Due to the near degeneracy of the two states, it would be extremely difficult in this case to detect the $\tau^{\prime}$ at LEP-2 through its dominant decay $\tau^{\prime} \rightarrow N_{\tau^{\prime}} W^{*}$, unless the $N_{\tau^{\prime}}$ decayed inside the collider detector.

\section{Phenomenological Restrictions and Implications}

In section 2, we noted that our model must possess a rather light top-squark (stop) whose mass is about $50 \mathrm{GeV}$. In order to achieve such a small $M_{\tilde{t}}$, there must be substantial $\tilde{t}_{L}-\tilde{t}_{R}$ mixing. $M_{\tilde{t}}^{2}$ is given by the smallest eigenvalue of the stop squaredmass matrix

$$
\left(\begin{array}{cc}
M_{Q}^{2}+m_{t}^{2}+c_{L} m_{Z}^{2} & m_{t}\left(A_{t}-\mu \cot \beta\right) \\
m_{t}\left(A_{t}-\mu \cot \beta\right) & M_{U}^{2}+m_{t}^{2}+c_{R} m_{Z}^{2}
\end{array}\right)
$$

where $M_{Q}, M_{U}$, and $A_{t}$ are soft-supersymmetry-breaking parameters, $c_{L} \equiv\left(\frac{1}{2}-\right.$ $\left.\frac{2}{3} \sin ^{2} \theta_{W}\right) \cos 2 \beta, c_{R} \equiv \frac{2}{3} \sin ^{2} \theta_{W} \cos 2 \beta$, and $\mu$ is the supersymmetric Higgs mass parameter. A light stop implies that the off-diagonal terms in Eq. (4.1) are of the same order as the geometric average of the diagonal terms,

$$
\left(m_{Q}^{2}+m_{t}^{2}\right)\left(m_{U}^{2}+m_{t}^{2}\right) \simeq m_{t}^{2} X_{t}^{2}
$$

where $X_{t} \equiv A_{t}-\mu \cot \beta$. This can be accomplished either by taking $X_{t}$ rather large or by pushing one of the soft supersymmetry breaking mass parameters to low values. However, the latter possibility is severely constrained by the constraints on the $\rho$ parameter and the bottom squark mass, as discussed in section 5 .

If there is large mixing in the third generation squark sector, one would naively expect that the fourth generation squark mixing would be large as well. If $X_{t}$, were of order $X_{t}$, the mixing in the fourth generation squark sector would be too large, driving the smallest eigenvalue of the $\tilde{t}_{L}^{\prime}-\widetilde{t}_{R}^{\prime}$ squared-mass matrix negative. Remarkably, due to the infrared fixed-point behavior of the fourth generation, $A_{t}$, is driven to a value that is independent of its high energy value [30]. Roughly, $A_{t^{\prime}} \simeq-1.5 m_{1 / 2}$ where $m_{1 / 2}$ is the high-energy (GUT-scale) value of the gaugino Majorana mass. In contrast, due to the smaller values of the top quark Yukawa coupling, the top squark mixing parameter $A_{t}$ is strongly dependent on its high energy value and hence can be taken as an independent parameter. Moreover, choosing $\mu$ negative and $A_{t}$ positive enhances the third generation squark mixing while somewhat suppressing the fourth generation squark mixing.

The constraints on the chargino and neutralino sectors provide information about the supersymmetric mass parameter $\mu$. If the gaugino Majorana mass parameters are unified with a common GUT-scale mass given by $m_{1 / 2}$, then the gluino, chargino and neutralino masses are determined by $m_{1 / 2}, \mu$, and $\tan \beta$. This is the situation in most grand unified theories (assuming that the threshold corrections, proportional to the GUT-symmetry breaking order parameter, are small). In order to obtain the desired 
region of parameter space where $m_{1 / 2} \ll|\mu|$ (with $\mu$ negative). Then, our choice of $M_{\tilde{\chi}_{1}^{0}} \simeq 25 \mathrm{GeV}$ fixes

$$
m_{1 / 2} \simeq \frac{\alpha\left(M_{\mathrm{GUT}}\right)}{\alpha_{2}\left(M_{Z}\right)} M_{2} \simeq 110 \mathrm{GeV} .
$$

In general, small threshold corrections at the grand unification scale will modify the exact relation among the gaugino masses. Assuming corrections of at most $25 \%$ in these relations, the typical values for the masses of the other light chargino and neutralino states are $M_{\tilde{\chi}_{1}^{ \pm}} \simeq M_{\tilde{\chi}_{2}^{0}} \simeq 55-70 \mathrm{GeV}$. The dominant decay of the chargino would be $\tilde{\chi}^{+} \rightarrow \tilde{t} \bar{b}$, followed by $\tilde{t} \rightarrow c \tilde{\chi}^{0}$. Note that the signature for this decay chain consists of bottom and charm quark initiated jets plus missing energy. In particular, such events would be phenomenologically distinct from the usual three body decays $\tilde{\chi}^{+} \rightarrow \tilde{\chi}^{0} W^{*}$ expected in the MSSM for a light gaugino-like chargino. The most recent data from LEP at $\sqrt{s}=136 \mathrm{GeV}$ places severe constraints on the chargino parameters of our model, but does not yet rule out the entire parameter space. The choice of $m_{1 / 2}$ also fixes the gluino mass; we find $M_{\tilde{g}} \simeq 150-200 \mathrm{GeV}$. The dominant decay of this gluino would be $\tilde{g} \rightarrow \tilde{t} \bar{t}$ (or its charge-conjugated state) followed by $\tilde{t} \rightarrow c \tilde{\chi}_{1}^{0}$. Such a gluino cannot be ruled out by present Tevatron limits.

The mass of the lightest CP-even Higgs boson should lie above the LEP lower limit. For $\tan \beta=1.6$, the tree-level upper bound on the light Higgs mass is $m_{h^{0}} \leq$ $m_{Z}|\cos 2 \beta| \simeq 40 \mathrm{GeV}$, which would have been detected at LEP. However, radiative corrections can substantially raise the tree-level upper bound [31]. In our model, the most important radiative corrections are associated with the fourth generation squark and quark masses. We employ the radiative corrections to the Higgs masses as outlined in Refs. [32-36]; it is crucial to include the complete fourth generation squark threshold effects, which depend on the off-diagonal squark mixing terms. For the values of $A_{t^{\prime}}$ and $A_{b}$ obtained from the infrared fixed point constraints and taking the $\mu$ parameter negative and of order the soft-supersymmetry-breaking diagonal squark mass parameters, we find that the experimental Higgs mass bound is fulfilled even for relatively low values of the fourth generation squark mass parameters, $m_{Q^{\prime}} \gtrsim 200 \mathrm{GeV}$. For example, for $m_{Q^{\prime}} \simeq 250 \mathrm{GeV}$, we find $m_{h^{0}} \simeq 65-70 \mathrm{GeV}$ as a typical range of values for the light CP-even Higgs mass, which is just above the present LEP limits.

\section{$5 \quad$ Precision Measurements and the Top Quark Mass}

Since the fourth generation quarks are relatively heavy and presumably are weakly mixed with the light quarks, their contributions to electroweak observables through virtual exchange occurs dominantly through loop corrections to the gauge boson selfenergies (i.e; the oblique corrections). In contrast, the third generation quark virtual effects are still responsible for the radiative corrections to $Z \rightarrow b \bar{b}$ and $b \rightarrow s \gamma$. However, the size of the virtual effects cannot be evaluated before specifying the value of the supersymmetric Higgs mass parameter $\mu$. 
$\Delta \rho$ be the one-loop contribution to $\rho$ arising from the various sectors of the supersymmetric model. Because the top quark mass is less than half of its standard value, the contribution of the $t-b$ doublet to $\Delta \rho$ is reduced by a factor of four. Since the fourth generation top quark mass $m_{t}$, is roughly of the same order as the standard top one, one might expect that the fourth generation fermions could make up the deficit in $\Delta \rho$. However, this is not the case due to the large value of $m_{b^{\prime}}$. Using

$$
\Delta \rho(t, b)=\frac{g^{2} N_{c}}{64 \pi^{2} m_{W}^{2}}\left[m_{t}^{2}+m_{b}^{2}-\frac{2 m_{t}^{2} m_{b}^{2}}{m_{t}^{2}-m_{b}^{2}} \log \left(\frac{m_{t}^{2}}{m_{b}^{2}}\right)\right],
$$

where $N_{c}=3$ is the color factor, it follows that the fourth generation fermions contribution to $\Delta \rho$ (for $m_{t^{\prime}}=175 \mathrm{GeV}$ and $m_{b^{\prime}}=105 \mathrm{GeV}$ ) is approximately the same as that from the third generation. Hence, the supersymmetric particle contributions must account for roughly a half of the total value of $\Delta \rho$. (Explicit formulae can be found in Appendix A.) This requirement places severe restrictions on the third and fourth generation squark mass parameters $M_{Q}, M_{U}$ and $M_{D}$ [see Eq. (4.1)]. One must maximize the off-diagonal squark mixing while keeping the diagonal squark mass parameters as small as possible. However, as discussed in section 4, the latter cannot be too small; otherwise the radiative corrections to the light Higgs mass will be reduced leading to a value of $m_{h^{0}}$ below the current LEP bound.

To obtain a sufficient squark contribution to $\Delta \rho$ is not a simple task. The squark contribution to $\Delta \rho$ arises only from loops involving $\tilde{t}_{L}$ and $\tilde{b}_{L}$. Since $m_{b} \ll m_{Z}$, there is very little mixing in the bottom squark sector, and it follows that $M_{\tilde{b}_{L}} \simeq M_{Q}$ should be larger than about $150 \mathrm{GeV}$. Furthermore, a large value of $M_{Q}$ implies that a significant squark contribution to $\Delta \rho$ may only come from large values of the off-diagonal squark mixing. Indeed, taking into account the requirement that $M_{\tilde{t}} \simeq 50 \mathrm{GeV}$, the breakdown of the custodial $S U(2)$ symmetry is best achieved by taking: (i) large values of the right handed squark mass parameter $\left(M_{U} \gg M_{Q}\right)$ to maximize the left handed component of the lightest stops; (ii) moderate or large values of $M_{Q}$ to maximize the mass difference between the left handed sbottom and the lightest stop; and (iii) large values of the off-diagonal stop mixing [as required by Eq. (4.2)]. The breakdown of the custodial symmetry arises predominantly as a result of the large squark mixing effects. By this procedure, we can make the stop contribution to the $\rho$ parameter as large as desired. However, the large hierarchy between the parameters $M_{U}$ and $M_{Q}$ and the large values of the mixing parameter $X_{t}$ needed to reproduce the observed value of the $\rho$-parameter seems rather ad-hoc. Thus, in what follows, we focus on a more natural choice of parameters where $M_{U}$ is of order $M_{Q}$, although large values of $X_{t}$ are required in this case as well.

There is a danger that large values of the mixing parameter $X_{t}$ may render the Standard Model vacuum state (globally) unstable [37,11,38]. In this case the true vacuum would break electric or color charge due to a non-zero vacuum expectation value of some squark or slepton field. However, the requirement of global stability of the electric and color charge conserving vacuum is perhaps too strong. The Standard Model vacuum can be metastable as long as its lifetime is sufficiently long relative to the age of the universe. The weaker condition of vacuum metastability can permit the 
The $\Delta \rho$ constraint essentially fixes the allowed range of soft-supersymmetry breaking diagonal squark masses. Consider first the third generation parameters. We take $M_{Q} \simeq M_{U}$, where the squark mixing parameter $X_{t}$ is chosen in such a way that the light stop has a mass fixed at $50 \mathrm{GeV}$. In this case, the contribution of the third generation squarks to $\Delta \rho$ is too small unless $M_{Q}$ lies above $200 \mathrm{GeV}$. The larger we take $M_{Q}$, the larger we must tune $A_{t}$ in order to preserve the light stop. This in turn raises $\Delta \rho$. For example, values of $m_{Q} \simeq 300 \mathrm{GeV}$ can generate a contribution from the third generation squarks which is of the same order as $\Delta \rho(t, b)$. However, in this case, one must also tolerate a rather large value of $X_{t} \simeq 1 \mathrm{TeV}$.

Non-negligible mixing in the fourth generation also enhances the fourth generation squark contributions to $\Delta \rho$. The maximum effect is limited by the lower bound on the mass of $\tilde{b}^{\prime}$. In order that $t^{\prime} \rightarrow b W^{+}$remain the dominant $t^{\prime}$ decay, one must kinematically forbid $t^{\prime} \rightarrow \tilde{b}^{\prime} \tilde{\chi}_{1}^{+}$. Given $M_{\tilde{\chi}_{1}^{ \pm}} \simeq 55-70 \mathrm{GeV}$, a value of $M_{\tilde{b}^{\prime}} \simeq 120 \mathrm{GeV}$ is a comfortable choice. Since $A_{t}$, and $A_{b}$, are determined by their infrared fixed point behavior, for a given value of the left and right handed squark mass parameters, the value of $\mu$ which maximizes the fourth generation squark contribution to $\Delta \rho$ can be obtained. All the phenomenological constraints have now forced the parameters of the model into a very narrow corner of parameter space.

It is convenient to parameterize the oblique radiative corrections in terms of the Peskin-Takeuchi variables [40] $S, T$ and $U$. Here $T \equiv \alpha^{-1} \Delta \rho$ (where $\alpha^{-1} \simeq 137$ ) is the most sensitive variable (although some interesting restrictions can be obtained by considering $S$ ). Langacker has performed a global analysis of precision electroweak data [41], assuming that $m_{t}=80 \mathrm{GeV}$ and $m_{h^{0}}=65 \mathrm{GeV}$, and extracts values for the oblique parameters. He finds $T_{\text {new }}=0.70 \pm 0.21$, which in our model must arise from the contribution of the fourth generation fermions and the third and fourth generation squarks. (The contributions from other supersymmetric particles are negligible.) Using Eq. (5.1), we find that the fourth generation fermions yield a contribution of 0.25 to $T_{\text {new }}$. The contributions of the third and fourth generation squarks depend sensitively on the squark parameters as noted above; a range of parameters can be found that yields a total squark contribution to $T_{\text {new }}$ between 0.3 and 0.4 . Due to the infrared fixed point relation, the fourth generation mixing mass parameters $A_{t^{\prime}} \simeq A_{b^{\prime}} \simeq-155 \mathrm{GeV}$. As an example, we find that if

$$
\begin{aligned}
m_{Q} \simeq m_{U} & \simeq 275 \mathrm{GeV} ; \quad \mu \simeq-460 \mathrm{GeV} \\
m_{Q^{\prime}} \simeq m_{U^{\prime}} & \simeq 250 \mathrm{GeV} \quad A_{t} \simeq 695 \mathrm{GeV}
\end{aligned}
$$

then the contribution from the third and fourth generation squarks to the $T$ parameter is approximately equal to 0.4 . Including the rest of the supersymmetric spectrum, we find $T_{\text {new }} \simeq 0.7$, which reproduces the central fitted value of $T_{\text {new }}$ quoted above. As for $S_{\text {new }}$, its value is still subject to large errors: $S_{\text {new }} \simeq-0.16 \pm 0.21$ in the analysis of Ref. [41]. Summing the contributions from the fourth generation fermions and light supersymmetric particles yields a small positive contribution to $S_{\text {new }}$. For example, typical model parameter choices yield a value of $S_{\text {new }} \simeq 0.2$, which lies within the $95 \%$ confidence level bound deduced from precision electroweak data. 
tive corrections to the $Z \rightarrow b \bar{b}$ vertex. The fourth generation effects are suppressed by mixing angles and are hence small. Due to the smaller top Yukawa coupling, the radiative corrections arising from the virtual exchange of third generation squarks are smaller than those of the MSSM for the same choice of chargino and stop masses. In addition, the large value of $|\mu|$ implies that the light chargino is nearly a pure gaugino. Thus, even though the chargino and stop masses are close to $\frac{1}{2} m_{Z}$, the effect of the chargino-stop loop has only a small effect on the rate $Z \rightarrow b \bar{b}$. Our model then predicts

$$
R_{b} \simeq 0.2184
$$

which, under the assumptions specified in section 2, is within one standard deviation of the measured LEP value [Eq. (2.3)]. The improvement over the Standard Model prediction is mainly due to the fact that $m_{t} \simeq m_{W}$.

The value of $R_{b}$ has also consequences for the value of $\alpha_{s}\left(M_{Z}\right)$ extracted at LEP. The value of $R_{\ell}$, from which $\alpha_{s}\left(M_{Z}\right)$ at LEP is extracted, would be different from the Standard Model value, due to the modification of $\Gamma_{\text {had }}$ induced through the larger value of $\Gamma_{b}$. In models where only the bottom-quark width of the $Z$-boson is modified by virtual corrections, a simple relation between $\alpha_{s}\left(M_{Z}\right)$ and $R_{b}$ may be obtained. In the one-loop approximation, it is given by

$$
\frac{\alpha_{s}\left(M_{Z}\right)}{\pi}=\left(\frac{1-R_{b}}{1-R_{b}^{0}}\right)\left(\frac{\alpha_{s}^{0}\left(M_{Z}\right)}{\pi}\right)-\frac{R_{b}-R_{b}^{0}}{1-R_{b}^{0}}
$$

where $\alpha_{s}^{0}\left(M_{Z}\right)=0.124 \pm 0.005$ is the value that would be obtained from the measured value of $R_{\ell}$ in the Standard Model (assuming $m_{h^{0}} \simeq 60 \mathrm{GeV}$ ), and $R_{b}^{0}=0.2156$ is the Standard Model value for $R_{b}$. Using the value of $R_{b}$ predicted by our model [Eq. (5.3)], we find $\alpha_{s}\left(M_{Z}\right) \simeq 0.112 \pm 0.005$, in good agreement with determinations of $\alpha_{s}$ from deep inelastic scattering data [42].

\section{Flavor Changing Neutral Current Processes}

The low value of the top quark mass can have far reaching consequences for flavor changing neutral current (FCNC) processes. We shall analyze the predictions for three particularly sensitive FCNC observables: the $b \rightarrow s \gamma$ decay rate, the $B_{d}-\bar{B}_{d}$ mixing and the CP-violation parameter $\epsilon_{K}[43]$. In our four generation supersymmetric model, the FCNC amplitudes depend not only on the top quark mass but also on the full set of supersymmetric parameters. In particular, the contribution of light stops and light charginos to these amplitudes can be quite significant. Moreover, the predictions depend strongly on the assumptions about the flavor structure of the softsupersymmetry-breaking parameters. As an indicative example, we shall assume that a super-GIM mechanism is active. In addition, we further assume that the mixing of the fourth generation with the first and second generations is negligibly small. We shall present the results for $M_{\tilde{\chi}^{ \pm}} \simeq 65 \mathrm{GeV}$ (the amplitudes considered below are slightly reduced for larger values of the chargino mass). The other model parameters are fixed by the phenomenological considerations of the previous sections. 
$b \rightarrow s \gamma$ is consistent with $1.0 \times 10^{-4} \lesssim \mathrm{BR}(b \rightarrow s \gamma) \lesssim 4 \times 10^{-4}$, as required by the CLEO measurement [44]. In Figure 4 we plot the prediction for the branching ratio $\mathrm{BR}(b \rightarrow s \gamma)$ in our model. It follows that, for values of the CP-odd Higgs mass $m_{A} \gtrsim 100 \mathrm{GeV}$, the prediction of our model lives comfortably within the experimental bound.

$\mathrm{BR}(\mathrm{b} \rightarrow \mathrm{s} \gamma) 10^{4}$

Figure 4. Branching ratio $\mathrm{BR}(b \rightarrow s \gamma)$ as a function of the Ce. $\mathrm{P}-$ od d mass $m_{A}$.

The bounds on $B_{d}-\bar{B}_{d}$ mixing and $\epsilon_{K}$ can place a lowe bound on the Standard Model top quark mass value. This bound can-be explicitly obtained from the following two equations taken from Ref. [45]. From the measurement of $\epsilon_{K}$, one deduces that:

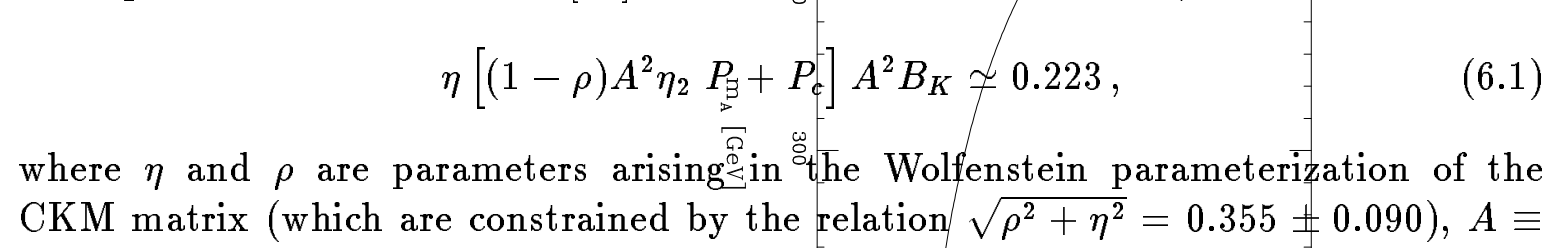
factor, $P$ is a reduced amplitude that arise $\bar{s}$ in the computation of the $W-t$ box diagram, $P_{c} \simeq 0.3$ is a small correction that arises from the box diagram mediated by the charmed quark, and $B_{K}=0.8 \pm 0.2$ represents the uncertainty in the hadronic matrix element.

g, one deduces that:

From the measurement of $B_{d}-\bar{B}_{d}$ mixing, one deduces that:

$$
\left|\frac{V_{t d}}{V_{u s} V_{c b}}\right|^{2}\left(\frac{0.75}{x_{d}}\right)\left(\frac{F_{B} \sqrt{B_{B}}}{200 \mathrm{MeV}}\right)^{2}\left|\frac{V_{c b}}{0.040}\right|^{2}\left(\frac{\tau_{B}}{1.6 \mathrm{ps}}\right)^{2} P \simeq 2.089,
$$

where $F_{B} \sqrt{B_{B}}=200 \pm 40 \mathrm{MeV},\left|V_{c b}\right|=0.040 \pm 0.003, \tau_{B}=1.6 \pm 0.1 \mathrm{ps}$, and $x_{d} \equiv$ $\Delta M_{B} / \Gamma_{B}=0.75 \pm 0.06$. 
tude $P$ depends on the top quark mass according to the following approximate formula:

$$
P_{\mathrm{SM}} \simeq 0.79\left(\frac{M_{t}}{m_{W}}\right)^{1.52}
$$

where $M_{t} \equiv m_{t}\left(m_{t}\right)$ is the $\overline{\mathrm{MS}}$ running top quark mass evaluated at the pole mass. For example, for a running mass $M_{t}=170 \mathrm{GeV}, P \simeq 2.465$. Scanning over the allowed values of all free parameters at the one sigma level we find

$$
0.8 \lesssim P \lesssim 10.5
$$

The lower bound on $P$ is relevant for our discussion. In particular, the lower bound on $P$ translates into a lower bound on the top quark mass given by $M_{t} \gtrsim 80 \mathrm{GeV}$.

We can now apply this analysis to any model for which an effective GIM mechanism is active. In particular, let $P_{4}$ be the value of $P$ obtained in our four-generation lowenergy supersymmetric model. We find that $P_{4}$ is enhanced with respect to $P_{\mathrm{SM}}$ for the same top quark mass, primarily due to the effects of light supersymmetric particle exchange. Figure 5 shows the ratio $P_{4} / P_{\mathrm{SM}}$ as a function of the CP-odd Higgs mass. We see that the enhancement factor is approximately 1.35 , with a dependence on $m_{A}$ an indication of the size of the charged Higgs exchange. Hence, the reduced amplitude in our model is $P_{4} \simeq 1.01$; which is within the one sigma bound quoted above. Note that this value strongly depends on our assumption about the presence of an effective GIM mechanism. If the model contains extra flavor violation parameters, the prediction for $P_{4}$ can be significantly modified. Furthermore, if new CP-violation phases are present in the theory, the above bounds on $P_{4}[\mathrm{Eq} .(6.4)]$ can be further relaxed. 


$$
\mathrm{P}_{4} / \mathrm{P}_{\mathrm{SM}}
$$

Figure 5. Ratio of the $B_{d}-\bar{B}_{d}$ reduced amplitude $P_{4}$ in the four generation model to the

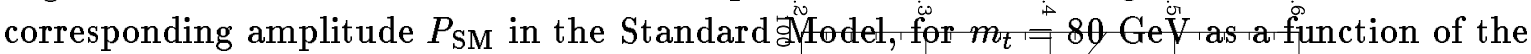
CP-odd Higgs mass $m_{A}$.

\section{Discussion and Conclusions :}

A low energy supersymmetric model wh four generations has been presented with $m_{t} \simeq m_{W}$, which satisfies all present day phënomenological constraints. Typical values of the model spectrum are shown in Table 1 . The fourth generation quark $t^{\prime}$ is identified as the state discovered by the CDF and Do Collaborations, while the light top quark decays via $t \rightarrow \tilde{t} \tilde{\chi}_{1}^{0}$, thereby escaping previous searches at hadron colliders. The oblique radiative corrections inferred from precision electroweak measurements at LEP are reproduced by contributions from the third and fourth generation quarks and squarks. The most theoretically troubling feature of the model is the large mixing among the third generation squarks that is necessary to ensure a light top squark and provide a viable prediction for the electroweak $\rho$-parameter.

There are several remarkable properties of the present model in contrast to the MSSM which should be stressed:

1. In the MSSM, any physics leading to larger values of $R_{b}$ also contributes to non-standard top quark decays [12]. Indeed, the potentially viable scenarios invoke either light charginos ${ }^{2}$ and light stops for small values of $\tan \beta$ and/or light charged Higgs bosons for large values of $\tan \beta$. Light charginos are usually associated with light neutralinos; hence, the decay mode $t \rightarrow \tilde{t} \tilde{\chi}^{0}$ is present, which can account for a significant fraction of the top quark branching ratio. As a result, in such models, the typical branching ratio of non-Standard Model top quark decays is of order $30 \%$.

\footnotetext{
${ }^{2}$ Based on the absence of light charginos in the most recent LEP run at $\sqrt{s}=136 \mathrm{GeV}, \mathrm{Ref}$. [13] quotes an absolute upper limit of $R_{b}<0.2174$ for the three-generation MSSM in the case of small $\tan \beta$.
} 
Table 1. Example of Model Spectrum and Parameters $(\tan \beta=1.6)$

\begin{tabular}{|lc|lc|}
\hline Particle & Mass $(\mathrm{GeV})$ & Particle & Mass $(\mathrm{GeV})$ \\
\hline$t$ & 80 & $\tilde{\chi}_{1}^{0}$ & 25 \\
$b^{\prime}$ & 105 & $\tilde{\chi}_{1}^{+}$ & 65 \\
$t^{\prime}$ & 175 & $\tilde{\chi}_{2}^{0}$ & 66 \\
$\tau^{\prime}$ & 50 & $\tilde{g}$ & 190 \\
$N_{\tau^{\prime}}$ & 80 & $\tilde{b}$ & 264 \\
$h^{0}$ & 67 & $\tilde{t}$ & 50 \\
$A^{0}$ & 300 & $\tilde{b}^{\prime}$ & 116 \\
$H^{ \pm}$ & 310 & $\tilde{t}^{\prime}$ & 263 \\
\hline \hline Parameter & Value $(\mathrm{GeV})$ & Parameter & Value $(\mathrm{GeV})$ \\
\hline$\mu$ & -460 & $M_{\tilde{Q}, \tilde{U}, \tilde{D}}$ & 275 \\
$M_{1}$ & 23 & $M_{\tilde{Q}^{\prime}, \tilde{U}^{\prime}, \tilde{D}^{\prime}}$ & 250 \\
$M_{2}$ & 54 & $A_{t, b}$ & 695 \\
$M_{3}$ & 170 & $A_{t^{\prime}, b^{\prime}}$ & -155 \\
\hline \multicolumn{4}{|c}{} \\
\hline
\end{tabular}

This is in slight disagreement with the Tevatron top decays observations, although the experimental errors are still large enough to permit a non-negligible reduction of $\mathrm{BR}\left(t \rightarrow b W^{+}\right)$from 1 . In our model, the decay of $t^{\prime} \rightarrow \tilde{t} \tilde{\chi}^{0}$ is a flavor violation process, which only occurs at the one loop level, and hence is naturally suppressed relative to the decay $t^{\prime} \rightarrow b W^{ \pm}$.

2. The large value of the top quark mass in the MSSM tends to lead to FCNC processes (which are particularly striking in the leptonic sector) induced by physics at the grand unification scale [46]. In our case, these effects are reduced due to the lower value of the top quark Yukawa coupling at $M_{\mathrm{GUT}}$ and the expected small mixing between the fourth and the first two generations.

3. The model presented in this paper exhibits the unification of the four fourth generation fermion-Higgs Yukawa couplings at a common high energy scale. In the MSSM, the unification of the bottom and top quark Yukawa couplings can only be obtained for very large values of $\tan \beta$, which usually implies a large fine tuning of the Planck scale parameters [47]. In contrast, $\tan \beta \sim \mathcal{O}(1)$ in our model, which is a more natural value for a model of bottom-top Yukawa coupling unification (and is certainly less fine-tuned than the large $\tan \beta$ model), especially given the proximity of the Yukawa couplings to their infrared fixed point values.

In a complementary work, Gunion, McKay and Pois [48] constructed four-generation models in the framework of minimal low-energy supergravity. The state discovered by the CDF and D0 Collaborations near $175 \mathrm{GeV}$ in mass was taken to be the top quark. Thus, to keep the fourth generation quark-Higgs Yukawa couplings perturbative up to 
In this alternative approach, the $R_{b}$ anomaly would have to be explained by assuming an appropriately light super-partner spectrum (as in the case of the three-generation MSSM). Gunion et al. also performed a complete renormalization group analysis of the soft-supersymmetry breaking terms in the four-generation models. In particular, they demonstrated that our model cannot be obtained from universal boundary conditions for the soft-supersymmetry-breaking parameters at $M_{\mathrm{GUT}}$. This result was due primarily to the large values of $A_{t}\left(M_{\mathrm{GUT}}\right)$ required to achieve a light top squark. Although helpful in suppressing flavor changing processes, the universality of scalar masses is not a necessary condition for obtaining an acceptable phenomenology. Even in the case of the three-generation MSSM, the violation of the universal boundary conditions seems to be a requirement for obtaining values of $R_{b}$ closer to the present experimental values $[7,11,49]$.

We have demonstrated that present day experimental data place severe constraints on the four-generation model parameters. Moreover, the model possesses a rich spectrum of new particles that will be accessible to LEP-2 and the Tevatron. In particular, eight new particles of this model could be discovered at LEP-2: the $t$-quark, the fourth generation leptons $\left(\tau^{\prime}\right.$ and $\left.N_{\tau^{\prime}}\right)$, the light Higgs boson $\left(h^{0}\right)$, and four supersymmetric particles $\left(\tilde{\chi}_{1}^{0}, \tilde{\chi}_{2}^{0}, \tilde{\chi}_{1}^{ \pm}\right.$, and $\left.\tilde{t}\right)$. New bounds coming from the initial run of LEP-2 at $\sqrt{s}=136 \mathrm{GeV}$ have constrained the model further. For instance, non-observation of the $\tau^{\prime}$ requires that it must be almost degenerate in mass with the fourth generation neutrino. Moreover, the non-observation of the lightest chargino pushes its mass to its upper allowed range, $M_{\tilde{\chi}^{ \pm}} \simeq 65-70 \mathrm{GeV}$. One can imagine scenarios where some of the properties of the eight particles listed above are altered to avoid conflict with future collider limits. ${ }^{3}$ However, it is certain that our model is ruled out unless a light top squark is discovered in LEP-2 data expected to be collected in 1996, or perhaps in the Run Ib data from the Tevatron after the complete data samples have been analyzed. Of course, if such a state is discovered, it could be consistent with the three-generation MSSM. In this case, the light top quark in our approach (or a fourth generation quark doublet with mass below that of the top quark in the approach of Gunion et al.) must be found to confirm the existence of a new generation. Alternatively, the exclusion of these models would raise ones confidence that the number of families in the effective

\footnotetext{
${ }^{3}$ As an example, if gaugino masses are not unified then the mass of $\tilde{\chi}_{1}^{ \pm}$is independent of the mass of the lightest neutralino if both states are dominantly gaugino-like (e.g., assuming $|\mu|$ is large compared to the gaugino mass parameters). Then, by choosing the $\tilde{\chi}_{1}^{ \pm}$mass above $100 \mathrm{GeV}$, one can tolerate a substantially lighter $\widetilde{b}^{\prime}$ than shown in Table 1, while still kinematically forbidding the two-body decay $t^{\prime} \rightarrow \widetilde{b}^{\prime} \tilde{\chi}_{1}^{+}$. (A lighter $\tilde{b}^{\prime}$ can be obtained by adjusting $\mu$ to generate larger mixing among the fourth generation squarks.) Three "benefits" ensue: (i) a lighter $\widetilde{b}^{\prime}$ can generate a larger fourth generation squark contribution to $\Delta \rho$ and increase the radiatively corrected value of $m_{h^{0}}$, thus allowing for less extreme mixing parameters in the third generation squark sector; (ii) the $b^{\prime}$ quark would be more difficult to detect at the Tevatron, since in this case $b^{\prime} \rightarrow \tilde{b}^{\prime} \tilde{\chi}_{1}^{0}$ followed by $\tilde{b}^{\prime} \rightarrow b \tilde{\chi}_{1}^{0}$ would be the dominant decay chain; and (iii) a heavier chargino would be kinematically inaccessible to LEP-2.
} 


\section{Acknowledgements}

We would like to thank Paul Langacker and Jens Erler for running their precision electroweak programs with $m_{t} \simeq m_{W}$, and for enlightening conversations. We are grateful to Ahmed Ali for encouraging us to take a more careful look at the implications of $\epsilon_{K}$ and $B-\bar{B}$ mixing, and to Guido Altarelli, George Hou, Mathias Neubert, and Sandip Pakvasa for a number of useful remarks. One of us (HEH) would like to acknowledge the support of the U.S. Department of Energy.

\section{Appendix A Squark contributions to the $\rho$-parameter}

This appendix contains the formulae for the squark contributions to the $\rho$-parameter. Only the contribution of the third generation squarks will be written out explicitly below. The contributions of the other squark generations can be trivially obtained by employing the appropriate squark masses and mixing angles (to a good approximation, squark mixing is appreciable only in the third and fourth generations). The formulae below can also be applied to the slepton contributions by setting the color factor ( $N_{c}=3$ for squarks) equal to unity.

We define the squark mixing angles as follows. Let $\tilde{q}_{1}$ and $\tilde{q}_{2}$ be the quark mass eigenstates, with $M_{\tilde{q}_{1}}<M_{\tilde{q}_{2}}$. (In this paper, we have denoted the light squark eigenstate $\tilde{q}_{1}$ by $\tilde{q}$, since the heavier squark eigenstate does not play an important role in our considerations.) Then, in terms of the interaction eigenstates $\tilde{q}_{L}$ and $\tilde{q}_{R}$,

$$
\begin{aligned}
& \tilde{q}_{1}=-\tilde{q}_{L} \sin \theta_{q}+\tilde{q}_{R} \cos \theta_{q}, \\
& \tilde{q}_{2}=\tilde{q}_{L} \cos \theta_{q}+\tilde{q}_{R} \sin \theta_{q} .
\end{aligned}
$$

For example, after diagonalizing the top-squark mass-squared matrix [Eq. (4.1)], one obtains

$$
\tan 2 \theta_{t}=\frac{2 m_{t}\left(A_{t}-\mu \cot \beta\right)}{M_{Q}^{2}-M_{U}^{2}+\left(c_{L}-c_{R}\right) m_{Z}^{2}} .
$$

The contribution of the third generation squarks to the $\rho$-parameter is then given by:

$$
\begin{aligned}
\Delta \rho(\tilde{t}, \tilde{b})=\frac{g^{2} N_{c}}{32 \pi^{2} m_{W}^{2}} & {\left[\sin ^{2} \theta_{t} \sin ^{2} \theta_{b} F\left(m_{\tilde{t}_{1}}^{2}, m_{\tilde{b}_{1}}^{2}\right)+\sin ^{2} \theta_{t} \cos ^{2} \theta_{b} F\left(m_{\tilde{t}_{1}}^{2}, m_{\tilde{b}_{2}}^{2}\right)\right.} \\
& +\cos ^{2} \theta_{t} \sin ^{2} \theta_{b} F\left(m_{\tilde{t}_{2}}^{2}, m_{\tilde{b}_{1}}^{2}\right)+\cos ^{2} \theta_{t} \cos ^{2} \theta_{b} F\left(m_{\tilde{t}_{2}}^{2}, m_{\tilde{b}_{2}}^{2}\right) \\
& \left.-\sin ^{2} \theta_{t} \cos ^{2} \theta_{t} F\left(m_{\tilde{t}_{1}}^{2}, m_{\tilde{t}_{2}}^{2}\right)-\sin ^{2} \theta_{b} \cos ^{2} \theta_{b} F\left(m_{\tilde{b}_{1}}^{2}, m_{\tilde{b}_{2}}^{2}\right)\right]
\end{aligned}
$$




$$
F\left(m_{1}^{2}, m_{2}^{2}\right) \equiv \frac{1}{2}\left(m_{1}^{2}+m_{2}^{2}\right)-\frac{m_{1}^{2} m_{2}^{2}}{m_{1}^{2}-m_{2}^{2}} \ln \left(\frac{m_{1}^{2}}{m_{2}^{2}}\right) \text {. }
$$

Note that in the supersymmetric limit $\left(M_{\tilde{t}_{1}}=M_{\tilde{t}_{2}}=m_{t}\right.$ and $\left.M_{\tilde{b}_{1}}=M_{\tilde{b}_{2}}=m_{b}\right)$, one finds $\Delta \rho(\tilde{t}, \tilde{b})=\Delta \rho(t, b)$ [see Eq. (5.1)]. In contrast, in the limit where $M_{Q}^{2} \gg m_{Z}^{2}$, $m_{t}^{2}$, the squark contribution to the $\rho$-parameter decouples:

$$
\Delta \rho(\tilde{t}, \tilde{b}) \simeq \frac{g^{2} N_{c} m_{t}^{4} K}{32 \pi^{2} m_{W}^{2} M_{Q}^{2}},
$$

where $K$ approaches a constant in the limit of large $M_{Q}^{2}$. One can evaluate $K$ by using the following properties of $F\left(m_{1}^{2}, m_{2}^{2}\right)$ in the limit of $\left|m_{1}^{2}-m_{2}^{2}\right| \ll m_{1}^{2}, m_{2}^{2}$

$$
\begin{aligned}
F\left(m_{1}^{2}, m_{2}^{2}\right) & \simeq \frac{\left(m_{1}^{2}-m_{2}^{2}\right)^{2}}{6 m_{2}^{2}} \\
F\left(m_{1}^{2}, m_{3}^{2}\right)-F\left(m_{2}^{2}, m_{3}^{2}\right) & \simeq\left(m_{1}^{2}-m_{2}^{2}\right)\left[\frac{1}{2}+\frac{m_{3}^{2}}{m_{3}^{2}-m_{2}^{2}}+\frac{m_{3}^{4}}{\left(m_{3}^{2}-m_{2}^{2}\right)^{2}} \ln \left(\frac{m_{2}^{2}}{m_{3}^{2}}\right)\right] .
\end{aligned}
$$

In this paper, it is the important third and fourth generation squark mixing effects that lead to a significant supersymmetric contribution to the $\rho$-parameter.

\section{References}

[1] F. Abe et al. [CDF Collaboration], Phys. Rev. Lett. 74 (1995) 2626; S. Abachi et al. [D0 Collaboration], Phys. Rev. Lett. 74 (1995) 2632.

[2] P. Antilogus et al. [LEP Electroweak Working Group], LEPEWWG/95-02 (1995).

[3] M. Veltman, Nucl. Phys. B123 (1977) 89.

[4] M. Boulware and D. Finnel, Phys. Rev. D44 (1991) 2054.

[5] A. Djouadi, G. Girardi, C. Verzegnassi, W. Hollik, and F. Renard, Nucl. Phys. B349 (1991) 48.

[6] G. Altarelli, R. Barbieri, and S. Jadach, Nucl. Phys. B369 (1992) 3; G. Altarelli, R. Barbieri and F. Caravaglios, Phys. Lett. B314 (1993) 357; Nucl. Phys. B405 (1993) 3.

[7] J.D. Wells, C. Kolda and G.L. Kane, Phys. Lett. B338 (1994) 219; G.L. Kane and J.D. Wells, SLAC-PUB-95-7038 (1995) [hep-ph/9510372].

[8] D. García, R.A. Jiménez and J. Solá, Phys. Lett. B347 (1995) 309; 321; [E: B351 (1995) 602]. 
Granlibakken, Tahoe City, CA, 13-18 December 1994, edited by J.F. Gunion, T. Han, and J. Ohnemus (World Scientific, Singapore, 1995) pp. 233-242.

[10] A. Dabelstein, W. Hollik and W. Mösle, Univ. of Karlsruhe preprint KA-THEP5-1995 [hep-ph/9506251].

[11] M. Carena and C.E.M. Wagner, Nucl. Phys. B452 (1995) 45

[12] X. Wang, J. Lopez and D.V. Nanopoulos, Phys. Rev. D52 (1995) 4116; E. Ma and D. Ng, TRI-PP-95-55 (1995) [hep-ph/9508338]; Y. Yamada, K. Hagiwara, and S. Matsumoto, KEK-TH-459 (1995) [hep-ph/9512227].

[13] J. Ellis, J.L. Lopez and D.V. Nanopoulos, CERN-TH/95-314 (1995) [hep$\mathrm{ph} / 9512288]$.

[14] H.E. Haber, CERN-TH/95-178 and SCIPP-95/31 (1995), to appear in the Proceedings of the XXXth Rencontre de Moriond, "Electroweak Interactions and Unified Theories", Les Arcs, Savoie, France, 11-18 March 1995.

[15] A. Blondel and C. Verzegnassi, Phys. Lett. B311 (1993) 346; J. Erler and P. Langacker, Phys. Rev. D52 (1995) 441; M. Shifman, Mod. Phys. Lett. A10 (1995) 605 .

[16] G.L. Kane, R.G. Stuart and J.D. Wells, Phys. Lett. B354 (1995) 350.

[17] D. García and J. Solá, Phys. Lett. B354 (1995) 335; B357 (1995) 349.

[18] F. Abe et al. [CDF Collaboration], Phys. Rev. Lett. 73 (1995) 220; Phys. Rev. D52 (1995) 2624.

[19] S. Abachi et al. [D0 Collaboration], Phys. Rev. Lett. 75 (1995) 1456.

[20] S. Abachi et al. [D0 Collaboration], Phys. Rev. Lett. 72 (1994) 2138.

[21] F. Abe et al. [CDF Collaboration] Phys. Rev. Lett. 73 (1994) 2667.

[22] G.W. Hou, Phys. Rev. Lett. 72 (1994) 3945.

[23] I.I. Bigi and S. Rudaz, Phys. Lett. B153 (1985) 335.

[24] Limits on supersymmetric particle masses are summarized in L. Montanet et al. [Particle Data Group], Phys. Rev. D50 (1994) 1173 and 1995 off-year partial update for the 1996 edition available on the PDG WWW pages (URL: http://pdg.lbl.gov/).

[25] S. Abachi et al. [D0 Collaboration], FERMILAB-CONF-95-187-E (1995), presented at the International Europhysics Conference on High Energy Physics, Brussels, Belgium, 27 July-2 August 1995. 
He and S. Pakvasa, Phys. Lett. 156B (1985) 236.

[27] S. Dimopoulos, S. Raby and F. Wilczek, Phys. Rev. D24 (1981) 1681; S. Dimopoulos and H. Georgi, Nucl. Phys. B193 (1981) 150; L. Ibañez and G.G. Ross, Phys. Lett. B105 (1981) 150.

[28] For a recent update and references to the earlier literature, see P. Langacker and N. Polonsky, Phys. Rev. D52 (1995) 3081; M. Carena, CERN-TH/95-319 (1995), presented at the International Europhysics Conference on High Energy Physics, Brussels, Belgium, 27 July-2 August 1995.

[29] C.T. Hill, Phys. Rev. D24 (1981) 691; C.T. Hill, C.N. Leung and S. Rao, Nucl. Phys. B262 (1985) 517.

[30] M. Carena, M. Olechowski, S. Pokorski, and C.E.M. Wagner, Nucl. Phys. B419 (1994) 213.

[31] H.E. Haber and R. Hempfling, Phys. Rev. Lett. 66 (1991) 1815; Phys. Rev. D48 (1993) 4280; Y. Okada, M. Yamaguchi and T. Yanagida, Prog. Theor. Phys. 85 (1991) 1; Phys. Lett. B262 (1991) 54; J. Ellis, G. Ridolfi and F. Zwirner, Phys. Lett. B257 (1991) 83; Phys. Lett. B262 (1991) 477; R. Barbieri, M. Frigeni, and F. Caravaglios Phys. Lett. B258 (1991) 167.

[32] R. Hempfling and A.H. Hoang, Phys. Lett. B331 (1994) 99

[33] J. Kodaira, Y. Yasui and K. Sasaki, Phys. Rev. D50 (1994) 7035

[34] J.A. Casas, J.R. Espinosa, M. Quirós and A. Riotto, Nucl. Phys. B436 (1995) 3; (E) B439 (1995) 466.

[35] M. Carena, J.R. Espinosa, M. Quiros and C.E.M. Wagner, Phys. Lett. B355 (1995) 209; M. Carena, M. Quiros and C.E.M. Wagner, CERN-TH/95-157 (1995) [hep-ph/9508343], Nucl. Phys. B, in press.

[36] H.E. Haber, R. Hempfling, and A.H. Hoang, CERN-TH/95-216 (1995).

[37] J.M. Frére, D.R.T. Jones and S. Raby, Nucl. Phys. B222 (1983) 11; J. Ellis, D.V. Nanopoulos and K. Tamvakis, Phys. Lett. B121 (1983) 123; L. Ibañez and C. Lopez, Phys. Lett. B126 (1983) 54; L. Alvarez-Gaumé, J. Polchinski and M. Wise, Nucl. Phys. B221 (1983) 495.

[38] P. Langacker and N. Polonsky, Phys. Rev. D50 (1994) 2199; J.A. Casas, A. Lleyda and C. Muñoz, FTUAM 95/11 (1995) [hep-ph/9507294].

[39] A. Kusenko, P. Langacker, and G. Segre, preprint in preparation.

[40] M.E. Peskin and T. Takeuchi, Phys. Rev. Lett. 65 (1990) 964; Phys. Rev. D46 (1992) 381. 
be found in J. Erler and P. Langacker, Phys. Rev. D52 (1995) 441; P. Langacker, NSF-ITP-95-140 and UPR-0683T [hep-ph/9511207], to appear in the Proceedings of the International Workshop on Supersymmetry and Unification of Fundamental Interactions (SUSY-95), Palaiseau, France, 15-19 May 1995.

[42] I. Hinchliffe, in the 1995 off-year partial update for the 1996 Particle Data Group edition available on the PDG WWW pages (URL: http://pdg.lbl.gov/).

[43] See, e.g., S. Bertolini, F. Borzumati, A. Masiero and G. Ridolfi, Nucl. Phys. B353 (1991) 591; R. Barbieri and G. Giudice, Phys. Lett. B309 (1993) 86; G.C. Branco, G.C. Cho, Y. Kizukuri and N. Oshimo, Nucl. Phys. B449 (1995) 483.

[44] M.S. Alam et al. [CLEO Collaboration], Phys. Rev. Lett. 74 (1995) 2885.

[45] A. Buras, Phys. Lett. B317 (1993) 449; MPI-PhT/95-88 (1995) [hep-ph/9509329], and references therein.

[46] L.J. Hall, V.A. Kostelecky, and S. Raby, Nucl. Phys. B267 (1986) 415; R. Barbieri and L.J. Hall, Phys. Lett. B338 (1994) 212; R. Barbieri, L.J. Hall, and A. Strumia, Nucl. Phys. B445 (1995) 219; Nucl. Phys. B449 (1995) 437.

[47] A.E. Nelson and L. Randall, Phys. Lett. B316 (1993) 516; L.J. Hall, R. Rattazzi, and U. Sarid, Phys. Rev. D50 (1994) 7048; M. Carena, M. Olechowski, S. Pokorski, and C. Wagner, Nucl. Phys. B426 (1994) 269; R. Rattazzi, and U. Sarid, SU-ITP94-16 and RU-95-13 (1995) [hep-ph/9505428].

[48] J.F. Gunion, D.W. McKay and H. Pois, Phys. Lett. B334 (1994) 339; UCD-95-18 (1995) [hep-ph/9507323].

[49] C.E.M. Wagner, CERN-TH/95-261 [hep-ph/9510341], to appear in the Proceedings of the International Workshop on Supersymmetry and Unification of Fundamental Interactions (SUSY-95), Palaiseau, France, 15-19 May 1995; M. Carena, P. Chankowski, S. Pokorski and C.E.M. Wagner, CERN-TH/95-302 (to appear). 